PROCEEDINGS OF THE

AMERICAN MATHEMATICAL SOCIETY

Volume 136, Number 7, July 2008, Pages 2299-2301

S 0002-9939(08)09263-0

Article electronically published on March 14, 2008

\title{
SUBGROUPS GENERATED BY SMALL CLASSES IN FINITE GROUPS
}

\author{
I. M. ISAACS
}

(Communicated by Jonathan I. Hall)

\begin{abstract}
Let $M(G)$ be the subgroup of $G$ generated by all elements that lie in conjugacy classes of the two smallest sizes. Avinoam Mann showed that if $G$ is nilpotent, then $M(G)$ has nilpotence class at most 3. Using a slight variation on Mann's methods, we obtain results that do not require us to assume that $G$ is nilpotent. We show that if $G$ is supersolvable, then $M(G)$ is nilpotent with class at most 3, and in general, the Fitting subgroup of $M(G)$ has class at most 4 .
\end{abstract}

\section{INTRODUCTION}

It was shown by N. Itô [2] in 1953 that finite groups having just two conjugacy class sizes must be nilpotent. Then for almost a half century, there was very little progress in understanding these groups until K. Ishikawa showed [1] that they have nilpotence class at most 3. Using a very much simpler proof, A. Mann [3] dramatically improved Ishikawa's result by showing that if $G$ is an arbitrary finite nilpotent group, then $M(G)$ has nilpotence class at most 3, where $M(G)$ is the subgroup generated by all elements of $G$ that lie in conjugacy classes of the two smallest sizes. Of course, if $G$ has just two class sizes, then $M(G)=G$, and since $G$ is nilpotent by Itô's theorem, Mann's result applies, and it shows that $G$ has nilpotence class at most 3. In other words, Ishikawa's theorem is an immediate consequence of Mann's.

In this note we present a slight simplification of Mann's argument, and this allows us to prove results for finite groups that are not necessarily nilpotent. For example, we obtain the following.

Theorem A. Let $G$ be a finite group that contains a normal abelian subgroup $A$ such that $\mathbf{C}_{G}(A)=A$. Then $M(G)$ is nilpotent, and it has nilpotence class at most 3 .

If $G$ is nilpotent, it is well known that every maximal abelian normal subgroup $A$ of $G$ satisfies $\mathbf{C}_{G}(A)=A$, and thus Theorem A applies and $M(G)$ has class at most 3. Mann's theorem, therefore, is included in our result. But also in supersolvable groups (and in certain other types of solvable groups, too) it is easy to show that $\mathbf{C}_{G}(A)=A$ for some abelian normal subgroup $A$. The following is thus immediate.

Received by the editors March 26, 2007.

2000 Mathematics Subject Classification. Primary 20D25.

Key words and phrases. Conjugacy class size, nilpotent, supersolvable, nilpotence class.

(C)2008 American Mathematical Society 
Corollary B. Let $G$ be finite and supersolvable. Then $M(G)$ is nilpotent with class at most 3 .

If we weaken the hypothesis in Corollary B and assume only that $G$ is solvable, it does not follow that $M(G)$ must be nilpotent. (The group $G=S L(2,3)$ provides an example, since $M(G)=G$ is solvable but not nilpotent.) We do obtain the following, however.

Theorem C. Let $G$ be a finite group and assume that $M(G)$ has a nonidentity solvable normal subgroup. Then $\mathbf{Z}(M(G))$ is nontrivial.

Finally, with no assumptions at all, we have the following theorem.

Theorem D. Let $G$ a finite group. Then the Fitting subgroup $\mathbf{F}(M(G))$ has nilpotence class at most 4 .

We have not been able to determine if the integer 4 in Theorem $\mathrm{D}$ can be reduced.

\section{THE KEY LEMMA}

All of our results rely on the following, which is a generalization of Mann's Theorem 1 in 3 . Mann's result is essentially the same as ours, except that he assumes that $G$ is a $p$-group, and his proof is somewhat more complicated.

Lemma 1. Let $K \triangleleft G$, where $G$ is an arbitrary finite group and $K$ is abelian. Let $x$ be a noncentral element of $G$, and let $y=[t, x]$ for some element $t \in K$. Then $\left|\mathbf{C}_{G}(y)\right|>\left|\mathbf{C}_{G}(x)\right|$, and so the $G$-class of $y$ is smaller than that of $x$.

Proof. Since $x$ is noncentral in $G$, the result is trivial if $y=1$, and so we assume that $y \neq 1$. Now let $H=K \mathbf{C}_{G}(x)$, and observe that $y=[t, x] \in K \subseteq H$. Of course, $\mathbf{C}_{H}(x)=\mathbf{C}_{G}(x)$, and so it suffices to show that $\left|\mathbf{C}_{H}(y)\right|>\left|\mathbf{C}_{H}(x)\right|$, or equivalently, that $\left|H: \mathbf{C}_{H}(y)\right|<\left|H: \mathbf{C}_{H}(x)\right|$.

If $u, v \in K$, we have $[u v, x]=[u, x]^{v}[v, x]=[u, x][v, x]$, where the second equality holds because $[u, x] \in K$ and $K$ is abelian. The map $\theta: K \rightarrow K$ defined by $\theta(k)=[k, x]$ is therefore a homomorphism, and so $\theta(K)$ is a subgroup, and $|\theta(K)|=$ $|K: \operatorname{ker} \theta|=\left|K: \mathbf{C}_{K}(x)\right|=\left|H: \mathbf{C}_{H}(x)\right|$. Now $K$ normalizes $\theta(K)$ because $K$ is abelian. Also, since $\theta(K)$ is uniquely determined by the normal subgroup $K$ and the element $x$, we see that it is normalized by $\mathbf{C}_{G}(x)$, and it follows that $\theta(K)$ is a normal subgroup of $K \mathbf{C}_{K}(x)=H$. But $y$ is a nonidentity element of $\theta(K)$, and thus the entire $H$-class $Y$ of $y$ consists of nonidentity elements of $\theta(K)$. Then $\left|H: \mathbf{C}_{H}(y)\right|=|Y|<|\theta(K)|=\left|H: \mathbf{C}_{H}(x)\right|$, as wanted.

Corollary 2. Let $K \triangleleft G$, where $G$ is an arbitrary finite group and $K$ is abelian. Then $[K, M(G)] \subseteq \mathbf{Z}(G)$.

Proof. If $x$ lies in a class of $G$ of size $m$, where $m$ is the smallest class size exceeding 1 , then by Lemma 1 , all elements of the form $[k, x]$ are central, where $k \in K$. The assertion is now immediate.

\section{Proofs}

We use the notation $G^{r}$ for the $r$ th term of the lower central series of $G$. Thus $G^{1}=G$, and by a result of P. Hall, $\left[G^{i}, G^{j}\right] \subseteq G^{i+j}$ for positive integers $i$ and $j$. 
Proof of Theorem $A$. We are given an abelian subgroup $A \triangleleft G$ with $\mathbf{C}_{G}(A)=A$, and we write $M=M(G)$. By Corollary 2, we have $[A, M] \subseteq \mathbf{Z}(G)$, and thus $[A, M, M]=1$. By the three-subgroups lemma, $M^{\prime} \subseteq \mathbf{C}_{G}(A)=A$, and thus $M^{4}=\left[M^{\prime}, M, M\right] \subseteq[A, M, M]=1$, and thus $M$ is nilpotent with class at most 3 .

As we indicated previously, Corollary B is immediate.

Proof of Theorem $C$. Again write $M=M(G)$. We are assuming that $M$ has a nontrivial solvable normal subgroup, and we want to show that $\mathbf{Z}(M)>1$. Let $Z=\mathbf{Z}(\mathbf{F}(M))$, so that $Z$ is a nontrivial characteristic abelian subgroup of $M$, and in particular, $Z \triangleleft G$. Then $[Z, M] \subseteq \mathbf{Z}(G)$ by Corollary 2, so if $[Z, M]>1$, then $\mathbf{Z}(G)$ is nontrivial. Since $\mathbf{Z}(G) \subseteq M$, we are done in this case. Otherwise, $[Z, M]=1$ and thus $1<Z \subseteq \mathbf{Z}(M)$.

Proof of Theorem D. Write $M=M(G)$ and $F=\mathbf{F}(M)$, and let $n$ be the nilpotence class of $F$, so that $F^{n}>1$ and $F^{n+1}=1$. Our goal is to show that $n \leq 4$, and so we can certainly assume that $n \geq 3$, and thus the subgroup $F^{n-2}$ is defined. If this normal subgroup of $G$ is abelian, then by Corollary 2 , we have $\left[F^{n-2}, M\right] \subseteq \mathbf{Z}(G)$, and thus $F^{n}=\left[F^{n-2}, F, F\right]=1$, which is a contradiction. Thus $F^{n-2}$ is nonabelian, and so $1<\left[F^{n-2}, F^{n-2}\right] \subseteq F^{2 n-4}$. Since $F^{n+1}=1$, we must have $2 n-4<n+1$, and thus $n<5$, as required.

\section{REFERENCES}

[1] K. Ishikawa, On finite $p$-groups which have only two conjugacy lengths. Israel J. Math. 129 (2002), 119-123. MR1910937 (2004b:20032)

[2] N. Itô, On finite groups with given conjugate types. I, Nagoya Math. J. 6 (1953), 17-28. MR0061597 (15:851c)

[3] A. Mann, Elements of minimal breadth in finite p-groups and Lie algebras. J. Austral. Math. Soc. 81 (2006), 209-214. MR2267792(2007i:20038)

Department of Mathematics, University of Wisconsin, 480 Lincoln Drive, Madison, WISCONSIN 53706

E-mail address: isaacs@math.wisc.edu 